\title{
Quantum-Mechanical Calculations in the Algebraic Group Theory
}

\author{
M. A. Olshanetsky
}

Institute of Theoretical and Experimental Physics, ITEP, SU-117259 Moscow, USSR

\begin{abstract}
Quantum oscillators on simple Lie algebras satisfying the special symmetry conditions are considered. Statsums, the Witten index and some simple correlators are calculated. The relations between these expressions and orders of algebraic groups over finite fields $\mathbb{F} q$ and degrees of some their representations are established under the condition that the temperature $T$ of systems is equal to $T=\omega / \ln q$. We consider the conformal limit of the theories where ranks of groups go to infinity. Also we discuss the relation between the adelic limit of the theories and the Tamagawa numbers.
\end{abstract}

\section{Introduction}

In the last several years methods of the quantum field theory were successfully applied in different domains of mathematics [1]. As a rule they allow to obtain the independent and transparent proofs of mathematical theorems. The aim of the present article is more modest - we give here only the quantum-mechanical interpretation of well-known formulae in the algebraic group theory. Nevertheless we hope that this approach leads to a deeper understanding of some relations in this subject.

The first part of the formulae concerns the orders of finite simple groups. The classification of these groups had been completed recently (see, for example [2]). The grand theorem states that the list of groups contains besides the alternating groups and groups of Lie type twenty six sporadic groups. In the present article we consider only the groups of Lie type over finite fields (GLT). Namely we establish the connection between the orders of GLT and statsums of quantum oscillators on simple Lie algebras over $\mathbf{R}$. More precisely statsums are calculated only for the part of GLT - the Chevalley groups. For the last groups - the so-called twisted groups - it is necessary to calculate the quantum average of twist operators. The connection appears when the temperature $T$ is equal to $\omega / \ln q$, where $\omega$ is a frequency of oscillators, $q=p^{r}$ and $p$ is a prime number. The similar situation 
occurs in the Dyson description of distribution of levels in heavy nuclei [3] - some fixed temperatures correspond to statistical systems on classical Lie groups. It is worthwhile to note also the analogy with the calculations of statsums in conformal field theories on a torus [4]. They depend on a parameter of a torus, i.e. on a temperature. In particular, if fields take values in a root lattice like in the heterotic string theory [5] or in a group manifold [6] then statsums are expressed through characters of representations of corresponding affine algebras [7]. Moreover in a field limit which exists for the groups of classical type over finite fields the statsums that we calculated here take the form of statsums on tori. From the different point of view we calculate the order of a group - the value of the character of regular representation on a unit element.

The generalisation of this construction is a calculation of characters of irreducible modular representations (representations in spaces over finite fields). These characters are known only in a few cases. For the groups $G L_{n}(q)$ they were calculated in [11] (see also [12]). It turns out that degrees of some of these representations (the values of characters on an unit element) coincide with the Witten index for harmonic oscillators with the special symmetry conditions. The fermions contributing in the index is nothing else but ghosts corresponding to some additional restrictions, imposed on systems.

This paper is organized as follows. In part 1 we present briefly the main facts about GLT and give the quantum-mechanical description of their orders. In Sect. 2 we first recapitulate the modular representations of the group $G L_{n}(q)$ and then discuss the quantum-mechanical interpretation of degrees of these representations. We also consider here the quantum systems related to the irreducible representation of the dihedral group. In Sect. 3 some additional issues are considered. First we derive formulae for the statsums when the ranks of groups tend to infinity. After an appropriate shifting of the vacuum energy the limiting statsums become modular forms on a torus. We also consider here similarities with the Dyson theory. The quantum systems with symmetries generated by complex reflections is a slight generalization of the systems under consideration. We discuss shortly this approach. In conclusion we consider an adelic ensemble of the quantum systems in a fermionic description: the fermionic oscillators with the "prime" frequencies $\omega_{p}=\ln p(p=2,3,5 \ldots)$. It is natural to suppose that there exists some special sigma-model, which incorporates this ensemble as an essential ingredient, with the distinguishing feature - the Witten index of the model is equal to the Tamagawa number of a simple group which is the symmetry group of the system.

\section{Quantum-Mechanical Calculations of Orders of GLT}

1. We give here a quick review of GLT. The original objects are a simple Lie algebra $\mathfrak{G}$ over $\mathbb{C}$ and a finite field $k$. There exists some special basis in $\mathbb{G}$ - the Chevalley basis - in which all structure constants are integer numbers. Let $R$ be a root system in $\mathbf{6}$. Then it is possible to choose generators $X_{\alpha}, \alpha \in R$ in a such way that the commutation relations in $\mathbb{G}$ have the following form:

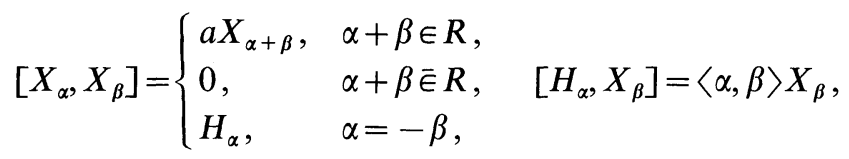


where $H_{\alpha}$ are Cartan generators. In simply laced algebras $a= \pm 1$. In other cases $a$ may also take values $\pm 2, \pm 3$. The Chevalley basis is generated by $\left\{X_{\alpha}, \alpha \in R\right\}$ and $\left\{H_{\alpha}\right.$ for simple roots $\left.\alpha\right\}$.

Let $V$ be a finite-dimensional representation of (5. In $V$ exists a lattice $M$ which is invariant under the action of all operators $X_{\alpha}^{m} / m !\left(\alpha \in R, m \in \mathbb{Z}^{+}\right)$. Note that the operators $X_{\alpha}$ are nilpotent. Therefore on the space $V^{k}=M \otimes_{\mathbb{Z}^{k}}$ the action of operators

$$
x_{\alpha}(t)=\exp \left(t X_{\alpha}\right)=\sum_{m=0}^{\infty} t^{m} X_{\alpha}^{m} / m !, \quad t \in k
$$

is well-defined. The operators $\left\{x_{\alpha}(t), t \in k\right\}$ generate a finite group GLT which is called the Chevalley group.

Consider, for example, the algebra $\left(\mathfrak{5}=s l_{2}\right.$ and the field $k=\mathbb{F}_{2}=\{0,1\}$. The Chevalley generators are $X_{ \pm \alpha}=\sigma^{ \pm}$and $H_{\alpha}=\sigma_{3}$. The Chevalley group generated by the matrices $X_{ \pm \alpha}(t)$ consists of the six matrices:

$$
\left(\begin{array}{ll}
1 & 0 \\
0 & 1
\end{array}\right),\left(\begin{array}{ll}
1 & 1 \\
0 & 1
\end{array}\right),\left(\begin{array}{ll}
1 & 0 \\
1 & 1
\end{array}\right),\left(\begin{array}{ll}
0 & 1 \\
1 & 0
\end{array}\right),\left(\begin{array}{ll}
0 & 1 \\
1 & 1
\end{array}\right),\left(\begin{array}{ll}
1 & 1 \\
1 & 0
\end{array}\right)
$$

This group is isomorphic to the permutation group $S_{3}\left(\left|S_{3}\right|=3\right.$ !) which is not simple - there is the normal subgroup of even permutations $A_{3}$ (the alternating group).

As a rule the Chevalley groups do not contain normal subgroups - it happens only for groups of lower ranks. But, generically the Chevalley groups do contain a center which has a following form $c=\operatorname{Hom}\left(L_{1} / L_{0}, k^{*}\right)$, where $L_{1}\left(L_{0}\right)$ is the weight (root) lattice in $\mathfrak{5}$ and $k^{*}$ is a multiplicative subgroup in $k$. Via factoring a largest "simply connected" group $G_{1}$ by $c$ we obtain the simple group $G_{0}$. The other examples of Chevalley groups:

1. The tetrahedron group $T \simeq A_{1}(3)=S l_{2}\left(\mathbb{F}_{3}\right)$.

2. The icosahedron group $I \simeq A_{1}(5)=S l_{2}\left(\mathbb{F}_{5}\right)$.

It is not the whole story about GLT - there exist also their twisted versions. These groups are constructed by means of external automorphisms of Lie algebras and automorphisms of fields. These automorphisms define the automorphism $\sigma$ of GLT $G$. The twisted group $G_{\sigma}$ is an invariant subgroup of $G$ under the action of $\sigma$. The automorphism $\sigma$ has the following description. Let $\varphi$ be an external automorphism of a Lie algebra 65 . It corresponds to an automorphism of the Dynkin diagram which generates a permutation $\varrho$ of roots $\{\alpha\}$ and generators $\left\{X_{\alpha}\right\}$. The automorphism $\varphi$ acts on the generators $x_{\alpha}(t)$ which has been earlier defined.

$$
\varphi\left(x_{\alpha}(t)\right)= \begin{cases}x_{\alpha \alpha}\left(\varepsilon_{\alpha} t\right) & \alpha \text { is a long root or } \mathfrak{G} \\ & \text { is a simply laced algebra } \\ x_{\alpha \alpha}\left(\varepsilon_{\alpha} t^{p}\right) & \alpha \text { is a short root }\end{cases}
$$

where $\varepsilon_{\alpha}= \pm 1$ ( $\varepsilon=1$ if $\alpha$ is a simple root), $p$ is the characteristic of $k$. It is worthwhile to note that if $p=2$ the algebras $C_{2}$ and $F_{4}$ have the external automorphism of order two. If $p=3 G_{2}$ also has the external automorphism of order two.

The automorphism $\sigma$ is defined as a joint action of $\varphi(1.1)$ and an automorphism $\theta$ of $k$. There are restrictions on an order of $\theta$. If $\varrho$ permutes roots 
with equal length then an order of $\varrho$ must be equal to an order of $\theta$ (and it is equal to 1,2 or 3). If $\varrho$ permutes unequal roots then $\gamma \theta^{2}=$ id, where $\gamma$ is the Frobenius automorphism $\gamma: t \rightarrow t^{p}(p$ is the characteristic of $k$ ).

Thus a twisted group $G_{\sigma}$ is the invariant subset

$$
G_{\sigma}=\{g \in G \mid \sigma g=g\} .
$$

The twisted groups are denoted as ${ }^{m} G_{l}(q)$ where $m$ is an order of $\sigma$. The twisted groups ${ }^{2} A_{n}(q),{ }^{2} D_{n}(q)(n>4),{ }^{2} E_{6}(q),{ }^{3} D_{4}(q)$ are called the Steinberg groups, the groups ${ }^{2} G_{2}(3),{ }^{2} F_{4}(2)$ are the Ree groups and the group ${ }^{2} C_{2}(2)$ is the Suzuki group. Note that any automorphism $\theta$ of $k$ is an element of cyclic group generated by $\gamma$. The order of this group is equal to $m$, where $q=p^{m}$ is the number of elements of $k$. Therefore for

$$
\theta: t \rightarrow t^{p^{a}}
$$

the following values of $q$ for different algebras are allowed

$$
\begin{gathered}
{ }^{2} A_{n}(q),{ }^{2} D_{n}(q),{ }^{2} E_{6}(q), \quad q=p^{2 a}, \\
{ }^{3} D_{4}(q), \quad q=p^{3 a}, \\
{ }^{2} G_{2}(q),{ }^{2} F_{4}(q),{ }^{2} C_{2}(q), \quad q=p^{2 a+1},
\end{gathered}
$$

2. We are interested in the formulae for orders of GLT. They are expressed through the degrees of invariant polynomials on $\mathfrak{G}$. Being restricted to Cartan subalgebras these polynomials generate the algebra of Weyl invariant polynomials $S^{W}$. The Chevalley-Shephard-Todd theorem (CSTT) describes this algebra $[8,9]$ :

Let $W$ be a finite group acting in a space $\mathfrak{h}$ and $S^{W}$ is defined as previously. Then

1. $S^{W}$ is generated by $n$ homogeneous polynomials $I_{1}, \ldots, I_{n}$, where $n=\operatorname{dimh}$.

2. The degrees $d_{1}, \ldots, d_{n}$ of the polynomials $I_{1}, \ldots, I_{n}$ are determined uniquely by $W$ (they are invariants of $W$ ).

3. If 1 is valid, then $W$ is generated by reflections.

All groups generated by reflections acting in a real space has been classified by Coxeter [10]. Beside the Weyl groups corresponding to Lie algebras these groups exist also in spaces of dimensions two $I_{2}(m)$ (dihedral groups), three $-H_{3}$ (icosahedral group) and four $-H_{4}$. The Coxeter groups and their invariants are written down in Table 1.

Let us present some needed relations. Let $N$ be a number of positive roots of $(\mathfrak{5}$. Then

$$
N=\sum_{i=1}^{n}\left(d_{i}-1\right) .
$$

The order of Coxeter group $W$ also can be expressed through invariants:

$$
|W|=\prod_{i=1}^{n} d_{i} .
$$

The dimension $f_{n}(\ell)$ of the space of homogeneous $W$-invariant polynomials of degree $\ell$ is equal to the number of solutions to the equation

$$
\ell=\sum_{i=1}^{n} \ell_{i} d_{i}, \quad \ell_{i} \geqq 0, \ell_{i} \in \mathbb{Z} .
$$


Table 1. The Coxeter groups

\begin{tabular}{lll}
\hline $\begin{array}{l}\text { Type of } \\
\text { groups }\end{array}$ & Orders of invariants & $N$ \\
\hline$A_{n}$ & $2,3, \ldots, n+1$ & $n(n+1) / 2$ \\
$B_{n}, C_{n}$ & $2,4, \ldots, n$ & $n$ \\
$D_{n}$ & $2,4, \ldots, 2(n-1), n$ & $n(n-1)$ \\
$G_{2}$ & 2,6 & 6 \\
$F_{4}$ & $2,6,8,12$ & 24 \\
$E_{6}$ & $2,5,6,8,9,12$ & 36 \\
$E_{7}$ & $2,6,8,10,12,14,18$ & 63 \\
$E_{8}$ & $2,8,12,14,18,20,24,30$ & 120 \\
$I_{2}(n)$ & $2, n$ & $n$ \\
$H_{3}$ & $2,6,10$ & 15 \\
$H_{4}$ & $2,12,20,30$ & 60 \\
\hline
\end{tabular}

The generating function for $f_{n}(\ell)$ (the Poincaré series of $S^{W}$ ) has the following form:

$$
F_{n}(x)=\prod_{j=1}^{n}\left(1-x^{d_{j}}\right)^{-1} .
$$

In [8] the formula for the orders of Chevalley groups is derived. It has the following form:

$$
|G(q)|=q^{N} \prod_{j=1}^{n}\left(q^{d_{j}}-1\right) .
$$

The orders of the twisted groups are calculated in the similar way. Considered previously the automorphism $\varrho$ generates the transformation of $\mathfrak{h}$ for which we conserve the notation $\varrho$. Let $\varepsilon_{j}$ be its eigenvalues $(j=1, \ldots, n)$. For all the twisted groups except ${ }^{3} D_{4} \varepsilon_{j}= \pm 1$. For ${ }^{3} D_{4} \varepsilon_{j}=\exp \frac{2 \pi i}{3} j(j=1,2,3), \varepsilon_{4}=1$. The orders of the twisted groups (1.2) are expressed through $\varepsilon_{j}$ and $d_{j}$,

$$
\left|G_{\sigma}(q)\right|=q^{N} \prod_{j=1}^{n}\left(q^{d_{j}}-\varepsilon_{j}\right) .
$$

The correspondence between $\varepsilon_{j}$ and $d_{j}$ will be pointed out further.

The formulae (1.6) and (1.7) give orders of simply connected versions. They are concretized in Table 2 . In the last column the orders $[c]$ of center $c$ are presented. An order of a simple group is equal to an order presented in Table 2 divided on $[c]$. Here $(a, b)$ is the common divisor of $a$ and $b$.

3. The quantum systems describe a motion of a quantum particle on a Cartan subalgebra $\mathfrak{h}=\mathbb{R}^{n}$ in an oscillator potential and thereto the states have a definite type of symmetry under the action of the Weyl group $W$. In the simplest case of $W$-invariant states it is possible to consider the enlarged configuration space - the whole algebra $\mathfrak{G}$ and correspondingly the invariant states on it. 
Table 2. The orders of GLT

\begin{tabular}{|c|c|c|c|}
\hline$G(q)$ & $\varepsilon_{j}$ & $|G(q)|$ & $|c|$ \\
\hline $\begin{array}{l}\text { The Chevalley } \\
\text { groups }\end{array}$ & 1 & $q^{N} \prod_{j=1}^{n}\left(q^{d_{j}}-1\right)(*)$ & $\left|\operatorname{Hom}\left(\frac{L_{1}}{L_{0}}, k^{*}\right)\right|$ \\
\hline${ }^{2} A_{n}$ & $(-1)$ & $q^{N} \prod_{j=1}^{n}\left(q^{d_{J}}-(-1)^{d_{J}}(* *)\right.$ & $(n+1, q+1)$ \\
\hline${ }^{2} D_{n}$ & $\begin{array}{l}-1 \text { for } d_{j}=n \\
1 \text { for other } d_{j}\end{array}$ & $\begin{array}{l}\text { in }(*) q^{n}-1 \\
\text { change on } q^{n}+1\end{array}$ & $\left(4, q^{n}\right)$ \\
\hline${ }^{3} D_{4}$ & $\begin{array}{l}1 \text { for } d_{j}=2,6 \\
\omega, \omega^{2} d_{j}=4,4 \\
\omega=\exp \frac{2 \pi i}{3}\end{array}$ & $\begin{array}{l}q^{12}\left(q^{2}-1\right)\left(q^{6}-1\right) \\
\left(q^{8}+q^{4}+1\right)\end{array}$ & 1 \\
\hline${ }^{2} E_{6}$ & $(-1)^{d_{J}}$ & $(* *)$ & $(3, q+1)$ \\
\hline${ }^{2} C_{2}$ & $\begin{array}{l}1 \text { for } d_{j}=2 \\
-1 \text { for } d_{j}=4\end{array}$ & $q^{4}\left(q^{2}-1\right)\left(q^{4}+1\right)$ & 1 \\
\hline${ }^{2} G_{2}$ & $\begin{array}{l}1 \text { for } d_{j}=2 \\
-1 \text { for } d_{j}=6\end{array}$ & $q^{6}\left(q^{2}-1\right)\left(q^{6}+1\right)$ & 1 \\
\hline${ }^{2} F_{4}$ & $\begin{array}{l}1 \text { for } d_{j}=2,8 \\
-1 \text { for } d_{j}=6,12\end{array}$ & $\begin{array}{l}q^{24}\left(q^{2}-1\right)\left(q^{6}+1\right) \\
\left(q^{8}-1\right)\left(q^{12}+1\right)\end{array}$ & 1 \\
\hline
\end{tabular}

We consider here the eigenvalue problem

$$
\begin{gathered}
H \Psi_{k}(x)=E_{k} \Psi_{k}(x), \quad x \in \mathfrak{h}, \\
H=\frac{1}{2} \sum_{j=1}^{n}\left(\hat{p}_{j}^{2}+\omega^{2} x^{2}\right), \quad \hat{p}_{j}=-i \partial / \partial x_{j}, \\
\int_{\mathfrak{h}}\left|\Psi_{k}(x)\right|^{2} d x<\infty .
\end{gathered}
$$

In this section we consider only the Hilbert space of invariant states

$$
\Psi(s x)=\Psi(x), \quad s \in W .
$$

It is not difficult to calculate the spectra of the systems. Introduce to this end the creation and the annihilation operators $a_{j}^{+}=\frac{1}{2}\left(\hat{p}_{j}+i \omega x_{j}\right), a_{j}=\frac{1}{2}\left(\hat{p}_{j}-i \omega x_{j}\right)$. Then $H=\sum_{j=1}^{n}\left\{a_{j}^{+}, a_{j}\right\}$ and

$$
\left[H,\left(a_{j}^{+}\right)^{m}\right]=m \omega\left(a_{j}^{+}\right)^{m} .
$$

Thus if $|k\rangle$ is an eigenstate with an energy $E_{k}$, then $\left(a_{j}^{+}\right)^{m}|k\rangle$ is also an eigenstate with an energy $E_{k}+m \omega$.

Let $I_{1}, \ldots, I_{n}$ be the complete system of generators of $S^{W}$. Consider operators $I_{1}\left(a_{1}^{+}, \ldots, a_{n}^{+}\right), \ldots, I_{n}\left(a_{1}^{+}, \ldots, a_{n}^{+}\right)$. As it follows from CSTT the action of these operators on the ground state

$$
|0\rangle=\exp \left(-\frac{\omega}{2} x^{2}\right), \quad E_{0}=\omega \frac{n}{2}
$$


gives the complete set of eigenstates

$$
|k\rangle=I_{1}^{k_{1}}, \ldots, I_{n}^{k_{n}}|0\rangle, \quad k=\left(k_{1}, \ldots, k_{n}\right)
$$

with energies [see (1.10)]

$$
E_{k}=E_{0}+\omega \sum_{j=1}^{n} k_{j} d_{j} .
$$

Now it is possible to calculate the statsum of the system

$$
Z(\beta)=\operatorname{Tr} \exp (-\beta H) \text {. }
$$

It follows from (1.12) that the degeneracy of the spectrum is given by the Poincare series (1.5). Therefore

$$
Z(\beta)=e^{-\beta E_{0}} \prod_{j=1}^{n}\left(1-e^{-\beta \omega d_{j}}\right)^{-1}
$$

Introducing the notation

$$
q=\exp \beta \omega \quad \text { or } \quad T=\omega / \ln q
$$

we come to the expression

$$
Z(q)=q^{-E_{0} / \omega} \prod_{j=1}^{h}\left(1-q^{-d_{J}}\right)^{-1}
$$

Note now, that the dimension of the algebra $(5$ is equal to $\operatorname{dim}(5=h+2 N$. Then taking into account (1.3) we express the statsum (1.16) through the orders of GLT (1.6):

$$
Z(q)=\frac{q^{-E_{0} / \omega+\operatorname{dim} \mathfrak{F}}}{|G(q)|} .
$$

After the appropriate shift of the Hamiltonian (1.8) $H^{\prime}=H+E^{\prime}$, where $E^{\prime}=-E_{0}+\omega \operatorname{dim}(5$, we come to the simple expression

$$
Z^{\prime}(q)=|G(q)|^{-1} \text {. }
$$

Consider now the twisted case. The group, generated by $\varrho$ acting in $\mathfrak{h}$, is a normalizator of $W$. Therefore $\varrho$ does not change invariant states (1.9). Recall now that either $\varrho^{2}=1$ or $\varrho^{3}=1$ and that the automorphism $\sigma$ defining $G_{\sigma}(1.2)$ is constructed by means of $\varrho$ and also depends on the characteristic $p$ of $k$. Consider the average of $\varrho$ in the Hilbert space

$$
\langle\varrho\rangle=Z^{\varrho}(q)=\operatorname{Tr}(\varrho \exp (-\varrho H)) \text {. }
$$

Operator $\varrho$ transforms a homogeneous invariant polynomial into itself

$$
\varrho I_{j}=\varepsilon_{j}^{0} I_{j}
$$

It was proved in [8] that $\varepsilon_{j}^{0}$ coincides with eigenvalues $\varepsilon_{j}$ of the operator $\varrho$ when it acts in $\mathfrak{h}: \varepsilon_{j}^{0}=\varepsilon_{j}$. Thereby the relation between $\varepsilon_{j}$ and $d_{j}$ in (1.7) is established. By means of $(1.12)\langle\varrho\rangle$ may be written as follows:

$$
Z^{\varrho}(q)=q^{-E_{0} / \omega} \prod_{j=1}^{n}\left(1-\varepsilon_{j} q^{d_{j}}\right)^{-1}
$$


Taking into account (1.3) and (1.7) we obtain the desired expression

$$
Z^{e}(q)=q^{-E_{0} / \omega+\operatorname{dim} \mathfrak{\sigma}}\left|G_{\sigma}(q)\right|^{-1} .
$$

Also as previously by shifting the vacuum energy $E_{0}$ we can get rid of the multiplier $q^{-E_{0} / \omega+\operatorname{dim} \omega}$.

\section{Degrees of Irreducible Modular Representations of $G L_{n}(q)$}

1. One of the main problems in the theory of GLT is a calculation of characters of irreducible representations in spaces $V^{d}$ over a field $k$ (modular representations). They are known only in a few cases. In particular for $G L_{n}(q)$ the complete table of characters has been constructed by Green $[11]$ (see also $[12,13])$. Here we write down the formulae for the degrees of irreducible representations of $G L_{n}(q)$ (the values of the characters on the unit element) and give the quantum-mechanical interpretation for some of them.

Let $k_{d}(d \geqq 1)$ be an algebraic extension of degree $d$ of $k$ and $k$ be its algebraic closure. Consider the set $\Phi=\{f\}$ of irreducible polynomials $f \in k[t]$, except $f(t)=t$, of degrees $d(f)$ with the highest coefficient 1 . The set of these polynomials can be described as follows. Let $x$ be a root of $f(t) \in \Phi$. Because the Frobenius automorphism $\gamma$ acting on roots of a polynomial over $k=\mathbb{F}_{q}$ does not change it, the roots of $f(t)$ can be obtained from $x$ by the action of $\gamma$ :

$$
\gamma^{r}(x)=\left(x^{q}\right)^{r}, \quad 0 \leqq r \leqq d-1, \quad f(t)=\prod_{i=0}^{d-1}\left(t-x^{q^{i}}\right) .
$$

Hence there is a one-to-one correspondence between roots of

$$
f(t):\left\{x, x^{q}, \ldots, x^{q^{d-1}}\right\}
$$

and the orbit of $\gamma$. Thus $\Phi$ is the set of orbits of $\gamma$ in $\bar{k}^{*}$.

Let for $f(t)=t^{d}-a_{d-1} t^{d-1} \ldots \pm a_{0} U(f)$ be a matrix of order $d$,

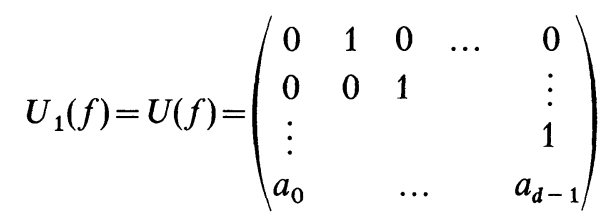

and for $m \geqq 1$,

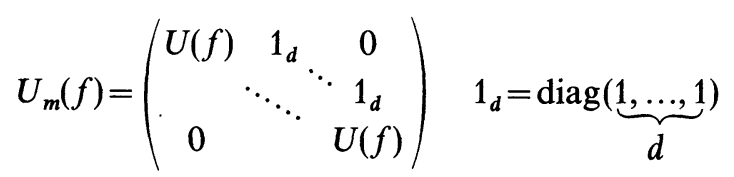

is a matrix built from $m$ diagonal blocks. If $\lambda$ is a partition of length $|\lambda|=k$ on $p$, positive integers $\ell_{1} \geqq \ldots \geqq \ell_{p}>0$ then the matrix

$$
U_{\lambda}(f)=\operatorname{diag}\left\{U_{\ell_{1}}(f), \ldots, U_{\ell_{p}}(f)\right\}
$$

has a characteristic polynomials $f(t)^{k}$. An arbitrary matrix $A \in G L_{n}(q)$ has a characteristic polynomial

$$
f=f_{1}^{k_{1}} \ldots f_{N}^{k_{N}}
$$


where $f_{1}, \ldots, f_{N}$ are irreducible polynomials of degree $d_{1}, \ldots, d_{N}$ over $k$ and $\sum k_{j} d_{j}=n$.

Any matrix $A \in G L_{n}(q)$ is conjugate to a block-diagonal matrix

$$
A \sim \operatorname{diag}\left\{U_{v_{1}}\left(f_{1}\right), \ldots, U_{v_{N}}\left(f_{N}\right)\right\}
$$

with a characteristic polynomial (2.2) with the restriction $\sum\left|v_{i}\left(f_{i}\right)\right| d\left(f_{i}\right)=n$. In other words a function $v(f)$ on the set of orbits $\Phi$ which takes values in partitions determines a conjugate class

$$
f_{1}^{v_{1}\left(f_{1}\right)} \ldots f_{N}^{\nu_{N}\left(f_{N}\right)}=\prod_{f \in \phi} f^{v(f)},
$$

which is represented by (2.3) with the additional restriction

$$
\sum_{f \in \phi} v(f) d(f)=n
$$

Consider as the simplest example the group $G L_{2}(q)$. The conjugated classes are represented by the matrices

$$
c_{j}=\left(\begin{array}{ll}
\alpha & 0 \\
0 & \alpha
\end{array}\right),\left(\begin{array}{ll}
\alpha & 1 \\
0 & \alpha
\end{array}\right),\left(\begin{array}{ll}
\alpha & 0 \\
0 & \beta
\end{array}\right),\left(\begin{array}{cc}
\eta & 0 \\
0 & \eta^{q}
\end{array}\right),
$$

where $\alpha, \beta \in \mathbb{F}_{q}^{*}$ (the multiplicative group of the field $\mathbb{F}_{q}$ ) $\eta, \eta^{q} \in \mathbb{F}_{q^{2}}^{*}$ are roots of an irreducible polynomial of degree 2 .

In accordance with (2.4) we have

$$
\begin{array}{ccc}
c_{1}=f^{2}, & \operatorname{deg} f=1, & v(f)=\{1,1\}, \\
c_{2}=f^{2}, & \operatorname{deg} f=1, & v(f)=\{2\}, \\
c_{3}=f_{1} f_{2}, & \operatorname{deg} f_{j}=1, & v\left(f_{j}\right)=\{1\}, \\
c_{4}=f, & \operatorname{deg} f=2, & v(f)=\{1\} .
\end{array}
$$

The degree of irreducible representation corresponding to a conjugate class $v(f)(2.4)$ has the following form. Consider a Young tableau for $v=\ell_{1}+\ldots+\ell_{p}$. Let $h(x)$ be a hook of a cell $x$ in a tableau - the sum of horizontal cells right from $x$ and lower vertical cells including $x$, and $f(x)$ be a foot - the sum of lower vertical cells. Then the degree of a representation is equal to

$$
\begin{gathered}
d_{v}(q)=\psi_{n}(q) \prod_{f \in \phi} q_{f}^{-|v|} \prod_{x \in \nu} \frac{q_{f}^{-f(x)}}{1-q_{f}^{-h(x)}}, \\
\sum_{f \in \phi} v(f) d(f)=n, \quad \psi_{n}(q)=(q-1) \ldots\left(q^{n}-1\right), \quad q_{f}=q^{\operatorname{deg} f} .
\end{gathered}
$$

For $G L_{2}(q)(2.6)$ takes the form

$$
d_{1}=1, \quad d_{2}=q, \quad d_{3}=q+1, \quad d_{4}=q-1 .
$$

Taking into account (2.5) we transform (2.6) to the form

$$
d_{v}(q)=q^{\frac{n(n+1)}{2}} \prod_{j=1}^{n}\left(1-q^{-j}\right) \prod_{x \in v(f)} \frac{q_{f}^{-f(x)}}{1-q_{f}^{-h(x)}} .
$$


For representation with $\operatorname{deg} f=1$ for all $f,(2.7)$ is simplified

$$
d_{v}(q)=q^{\frac{n(n+1)}{2}} \prod_{j=1}^{n}\left(1-q^{-j}\right) \prod_{x \in v} \frac{q^{-f(x)}}{1-q^{-h(x)}} .
$$

2. Here we present a quantum-mechanical interpretation of $d_{v}(q)(2.8)$. To this end it is natural to consider a quantum system on $g l_{n}$, or excluding an independent degree of freedom related to a trace of a matrix - a system on $s l_{n}$. In fact, we consider a generic case - a system related to an arbitrary Coxeter group (including a noncrystallographic one).

In this connection it is necessary to change the boundary conditions (1.2). Let the wave-functions carry an additional index which enumerates a basis of an irreducible representation $v$ of Coxeter group ${ }^{1}$

$$
T^{(v)}(s) \Psi^{(\nu)}(x)=\left.T^{(v)}\right|_{a} ^{b} \Psi_{b}^{(\nu)}(x)=\Psi^{(\nu)}(s x) .
$$

Because a Coxeter group $W$ is generated by reflections which commute with the Hamiltonian (1.8), the wave-functions $\Psi^{(v)}(s x)$ correspond to the same energy level for all $s \in W$. Let $R_{+}$be a set of positive roots and $A=\{x \in \mathfrak{h} \mid(x, \alpha) \geqq 0\}$ be a positive Weyl chamber. Then by means of (2.9) it is possible to restrict $\Psi(x)$ to $\Lambda$ with appropriate boundary conditions on walls $(x, \alpha)=0$ of $\Lambda$.

Note also that an arbitrary wave-function has a form

$$
\Psi_{k}(x)=I_{k}(x)|0\rangle,
$$

where $I_{k}(x)$ is a multidimensional analog of the Hermite polynomials which satisfies the equation

$$
\left[\Delta-2(x, \partial)+2\left(E_{k}-E_{0}\right)\right] I_{k}(x)=0
$$

and the symmetry conditions which follow from (2.9).

The simplest conditions (2.9) correspond to semi-invariants [9] which are defined by means of unitary characters $\chi^{(v)}$ of the Coxeter groups

$$
\Psi^{(v)}(s x)=\chi^{(v)}(s x) \Psi^{(v)}(x), \quad s \in W .
$$

If $\chi^{v}(s) \equiv 1$ is the trivial character, then the states $\Psi^{(v)}$ are invariant ones. The antiinvariant states correspond to the character $\chi^{(v)}(s)=\operatorname{det} s$. To calculate their ground-state one must use (2.10), (2.11),

$$
|0\rangle^{\mathrm{ant}}=\prod_{\alpha \in R_{+}}(\alpha, x)|0\rangle^{\mathrm{inv}} .
$$

Here $|0\rangle^{\text {inv }}$, is given by (1.11) and $N$ is a number of positive roots. The simplylaced algebras $A, D, E$ have no other semi-invariants. For $B, C, G_{2}, F_{4}$ algebras there are two additional semi-invariants. Let $R_{+}^{\ell}, R_{+}^{\text {sh }}$ be subsets of long and short roots in $R_{+}: R_{+}=R_{+}^{\ell} \cup R_{+}^{\text {sh }}$. These subsets define the corresponding semi-invariant ground-states

$$
\begin{aligned}
|0\rangle^{\ell}=\prod_{\alpha \in R_{+}^{l}}(\alpha, x)|0\rangle^{\mathrm{inv}}, & E_{0}^{\ell}=E_{0}^{\mathrm{inv}}+N_{\ell} \omega, \\
|0\rangle^{\mathrm{sh}}=\prod_{\alpha \in R_{+}^{\mathrm{sh}}}(\alpha, x)|0\rangle^{\mathrm{inv}}, & E_{0}^{\mathrm{sh}}=E_{0}^{\mathrm{inv}}+N_{\mathrm{sh}} \omega .
\end{aligned}
$$

\footnotetext{
* Irreducible representations of crystallographic groups are listed in [14]
} 
A Coxeter group $W$ acts transitively on subsets of roots of equal length. Thus the semi-invariant ground-states one-to-one correspond to the set of orbits of $W$ in a set $R$ of roots: 1) invariant states $|0\rangle^{\text {inv }} \rightarrow a$ trivial orbit 2) $|0\rangle^{1} \rightarrow R^{1}$ 3) $|0\rangle^{\mathrm{sh}} \rightarrow R^{\mathrm{sh}}$, 4) $|0\rangle^{\text {ant }} \rightarrow R^{\text {sh }} \cup R^{1}=R$.

An arbitrary semi-invariant is obtained from the semi-invariant ground-states by the multiplication on a polynomial from $S^{w}$ [see (2.10)].

For the quantum systems of type $A_{n-1}$ which are our main interest the Coxeter group is isomorphic to the permutation group $S_{n}$. The quantum system describes a one-dimensional $n$-body problem. The invariant (anti-invariant) wave-functions describe bosons (fermions). More general conditions (2.9) correspond to parastatistic wave-functions. Consider an irreducible representation $v$ of $S_{n}$ which is defined by an Young tableau $v=\left\{n_{1}, \ldots, n\right\}$, where $|v|=n=n_{1}+\ldots+n_{k}$, $n_{1} \geqq n_{2} \geqq \ldots \geqq n_{k}>0$. For example, for the invariant state $v=\{n\}$ and for the antiinvariant $v=\{1, \ldots, 1\}=\left\{1_{n}\right\}$. The dimension of a space of an irreducible representation $v$ is given by the "hook" formula [12]:

$$
\operatorname{dim} v=\frac{n !}{\prod_{x \in v} h(x)},
$$

where $h(x)$ is the hook of a cell $x$.

Return now to the general situation. In addition to (2.9) we put also the following constraints: any state is annihilated by the all $W$-invariant combinations of the annihilation operators:

$$
\begin{gathered}
P\left(a_{1}, \ldots, a_{n}\right) \Psi^{(v)}(x)=0 ; \\
P \in S^{W} .
\end{gathered}
$$

Let $S(\mathfrak{h})$ be an algebra of polynomials on $\mathfrak{h}$ generated by the creation operators $a_{1}^{+}, \ldots, a_{n}^{+}$. For free oscillators the ground state will be a symmetric one, $|0\rangle^{\text {inv }}$ (1.11), and the functions

$$
\begin{gathered}
|k\rangle=a_{1}^{+k_{1}} a_{2}^{+k_{2}} \ldots a_{n}^{+k_{n}}|0\rangle^{\text {inv }}, \quad k_{j} \geqq 0, k_{j} \in \mathbb{Z}, \\
k=\left(k_{1}, \ldots, k_{n}\right),
\end{gathered}
$$

in accordance with (1.10) are eigenstates with the energy

$$
E_{k}=E_{0}^{\mathrm{inv}}+\omega \sum_{j=1}^{n} k_{j}
$$

The energy $E_{k}$ defines the standard gradation of the polynomial algebra $S(\mathfrak{h})$. Thus the statsum for free oscillators has the form

$$
Z(q)=\operatorname{Tr}\left(e^{-\beta H}\right)=\frac{q^{-\frac{n}{2}}}{\left(1-q^{-1}\right)^{n}}, \quad q=e^{\beta \omega} .
$$

Restrictions on creation operators in (2.18) are reflected in the highest degree of the polynomials $I_{k}(x)$ in (2.10). Namely, the application $a_{j}^{+}$in $(2.18)$ is equivalent to multiplication on $x_{j}$ in (2.10). Similar, the annihilation by $a_{j}$ is equivalent to the differentiation $\partial_{j}$. Consider for example the free oscillators with the constraints 
(2.17). Put now under $\operatorname{Tr}$ the operator $(-1)^{F}$ which gives the difference between the number of boson and fermion states. The last are generated by the fermion ghosts corresponding to the constraints (2.17). In fact we go from the statsum to the Witten index. Taking into account the last remark and comparing (1.16) and (2.17)-(2.19) we obtain

$$
W(q)=\operatorname{Tr}(-1)^{F} e^{-\beta H}=\frac{q^{-\frac{n}{2}} \prod_{j=1}^{n}\left(1-q^{-d_{j}}\right)}{\left(1-q^{-1}\right)^{n}}=q^{-\frac{n}{2}} P\left(q^{-1}\right)
$$

$P\left(q^{-1}\right)$ is a polynomial of degree $N$. Hence only on a finite number of levels the operator $(-I)^{F}$ does not vanish. The energy of the highest level is equal to $E_{0}+\omega N$ $=\omega\left(\frac{n}{2}+N\right)$. The polynomial $P(q)$ has an interesting geometrical description in terms of an action of the Coxeter group $[8,15]$.

To build the states satisfying also (2.9) it is necessary to apply to the ground state $|0\rangle^{\text {inv }}$ in (2.18) a homogenous polynomial which is transformed in accordance with (2.9). In view of (2.17) it is necessary to consider only polynomials which are annihilated by all $W$-invariant combinations of the differentiations $\partial_{1}, \ldots, \partial_{n}$. Denote the dimensions of spaces of these polynomials as $M_{k}^{v}$, where $k$ is their degree. The Poincaré series

$$
P_{v}\left(q^{-1}\right)=\sum_{r=0}^{\infty} M_{r}^{v} q^{-r}
$$

for the classical Coxeter groups is calculated in [15]. In particular, for the Coxeter group of type $A_{n-1}$ which corresponds to the Lie algebra $g l_{n}$ it takes the form

$$
p_{v}\left(q^{-1}\right)=\prod_{k=1}^{n}\left(1-q^{-k}\right) \frac{\prod_{k=1}^{n} q^{-f_{k}}}{\prod_{k=1}^{n}\left(1-q^{-h_{k}}\right)} .
$$

Thus the Witten index for the systems satisfying (2.9) and (2.17) is equal to

$$
W(q)=q^{-n / 2} \prod_{k=1}^{n}\left(1-q^{-k}\right) \prod_{k=1}^{n} \frac{q^{-f_{k}}}{1-q^{-h_{k}}},
$$

or, as it follows from (2.8),

$$
W(q)=q^{\frac{n(n+2)}{2}} d_{v}(q),
$$

where $d_{v}(q)$ is the degree of representation with $\operatorname{deg} f=1$. Note that nontrivial energy levels vary between

$$
E_{\min }^{(v)}=E_{0}^{\mathrm{inv}}+\omega \sum_{k=1}^{n} f_{k}
$$

and

$$
E_{\max }^{(v)}=E_{0}^{\mathrm{inv}}+\omega\left[\frac{n(n+1)}{2}+\sum_{k=1}^{n}\left(f_{k}+h_{k}\right)\right]
$$


Shifting the Hamiltonian $H \rightarrow H+E^{\prime}$, where $E^{\prime}=\omega \frac{n(n+2)}{2}$, we reproduce (2.8) exactly. In fact, it is possible to give a similar description of the general formula (2.7). To this end it is necessary to modify the definition of annihilation and creation operators. We shall not dwell on this issue.

3. As we mentioned before the formulae of type (2.22) have been derived for the all classical groups in [15]. Here we consider the dihedral group $I_{2}(n)$. The corresponding system describes a quantum particle in the angle $2 \pi / n$ in the harmonic potential and the continuation of wave-functions on the whole plane $\mathbb{R}^{2}$ is implemented by (2.9), where $v$ is an irreducible representation of $I_{2}(n)$. The wave functions corresponding to the invariant and anti-invariant conditions were calculated exactly in [16]. Here we calculate the Witten index for these systems with the constraints (2.17). For free oscillators it takes the form (see Table 1)

$$
W(q)=q^{-1} \frac{\left(1-q^{-2}\right)\left(1-q^{-n}\right)}{\left(1-q^{-1}\right)^{2}}
$$

The group $I_{2}(n)$ has one-dimensional and two-dimensional irreducible representations. The semi-invariants correspond to the one-dimensional representations. The decomposition of the regular representation of $I_{2}(n)$ which has dimension $2 n$ takes the different forms for the even and odd $n$. Namely

$$
\begin{gathered}
n=2 m, \quad \underline{4 m}=4 \cdot 1+2 \cdot \sum_{i=1}^{m-1} 2^{(i)}, \\
n=2 m+1, \quad \underline{2(2 m+1)}=2 \cdot 1+2 \sum_{i=1}^{m} 2^{(i)} .
\end{gathered}
$$

In correspondence with (2.25) the Witten index has the decomposition on the irreducible components:

$$
\begin{aligned}
\operatorname{Tr} & (-1)^{F} e^{-\beta H} \\
& =\left\{\begin{array}{lr}
q^{-1}+2 \cdot q^{-m-1}+q^{-2 m-1}+2 \cdot\left\{q^{-2}\left(1+q^{-n}\right)+\ldots+q^{-m}\left(1+q^{-n}\right)\right\} & n=2 m \\
q^{-1}+q^{-2 m-2}+2 \cdot\left\{q^{-2}\left(1+q^{-1}\right)+\ldots+q^{-2 m}\left(1+q^{-1}\right)\right\} . & n=2 m+1
\end{array}\right.
\end{aligned}
$$

Every summand here corresponds to the Witten index of a system in which wavefunctions are transformed in an irreducible one-dimensional or two-dimensional representation $v$ of $I_{2}(n)$.

\section{Comments}

1. It is possible to consider a field theoretical limit $n \rightarrow \infty$ for the quantum systems related to the algebras of classical types. Let us replace in (1.20)

Then in (1.19),

$$
q=\exp (-2 \pi i \tau), \quad \tau=-\frac{i \beta \omega}{2 \pi}
$$

$$
Z^{\varrho}(\tau)=\frac{e^{-2 \pi i \tau \frac{E_{0}}{\omega}}}{\prod_{j=1}^{n}\left(1-\varepsilon_{j} e^{-2 \pi i \tau d_{j}}\right)}
$$


Consider the $\lim _{n \rightarrow \infty} Z^{e}$. In this limit the vacuum energy $E_{0}(1.11)$ is diverged, and we use below the zeta-function regularisation: $\lim _{n \rightarrow \infty} n=-\frac{1}{2}$.

Consider the transformation properties of the limit under the modular transformations generated by $T: \tau \rightarrow \tau+1$, and $S: \tau \rightarrow-1 / \tau$. In some cases a limit statsum is transformed as an element of finite-dimensional representation of the modular group in a space of modular functions with a fixed weight. After the regularization and an appropriate shift of a Hamiltonian $H \rightarrow H+E^{\prime}$ one obtains

$$
\begin{gathered}
\lim _{n \rightarrow \infty} Z_{A_{n}}=Z_{A}=\eta^{-1}(\tau), \\
\lim _{n \rightarrow \infty} Z_{B_{n}}=\lim _{n \rightarrow \infty} Z_{C_{n}}=Z_{B}=\eta^{-1}(2 \tau), \\
\lim _{n \rightarrow \infty} Z_{(2) A_{n}}=Z_{(2) A}=\left[\eta(2 \tau) \theta_{3}(2 \tau)\right]^{-1 / 2} .
\end{gathered}
$$

Here $\eta(\tau)=q^{1 / 24} \prod_{j=1}^{\infty}\left(1-q^{j}\right)$ is the Dedekind function,

$$
\theta_{3}(\tau)=\prod_{j=1}^{\infty}\left(1-q^{j}\right)\left(1+q^{j-1 / 2}\right)^{2}
$$

is the theta-function. In the first expression we include the invariant $d_{1}=I$ in the algebra $S^{w}$, i.e. we replace the group $S L_{n}(q)$ on the group $G L_{n}(q)$. This scheme has failed for the $D_{n}$ systems due to the additional invariant of order $n$. It is not difficult to establish that the modular group acts on the limit statsums as follows:

$$
\begin{gathered}
T\left(Z_{A}\right)=e^{-\frac{i \pi}{12}} Z_{A}, Z\left(Z_{A}\right)=(-i \tau)^{-1 / 2} Z_{A}, \\
T\left(Z_{B}\right)=e^{-\frac{i \pi}{6}} Z_{B}, Z\left(Z_{B}\right)=(-2 i \tau)^{-1 / 2} Z_{B}, \\
T\left(Z_{(2)_{A}}\right)=e^{-\frac{i \pi}{12}}\left(\eta(2 \tau) \theta_{4}(2 \tau)\right)^{-1}, \\
Z\left(Z_{(2)_{A}}\right)=(-2 i \tau)^{-1 / 2} Z_{(2) A},
\end{gathered}
$$

where $\theta_{4}(\tau)=\prod_{j=1}^{\infty}\left(1-q^{j}\right)\left(1-q^{j-1 / 2}\right)^{2}$.

2. Now consider the quasiclassical limit of the statsum (1.20). We replace $\beta \omega$ by $\hbar \beta \omega$ and tend $\hbar \rightarrow 0$. Thus this limit is equivalent to the high temperature limit $T \rightarrow \infty$, or $\omega \rightarrow 0$. From (1.15), we immediately obtain

$$
Z_{\mathrm{cl}} \sim \frac{1}{(\beta \omega)^{n} \prod_{j=1}^{n} \varepsilon_{j} d_{j}}
$$

or, taking into account that $\prod_{j=1}^{n} \varepsilon_{j}= \pm 1$ and (1.4),

$$
Z_{\mathrm{cl}} \sim \frac{1}{(\beta \omega)^{n}|W|}
$$


Comparing this expression with (1.20) we can conclude that in the high temperature limit the statsum "knows" only about the original $W$-symmetry. Then after cooling it looks like the symmetry is "increased" in the "integer" points $T=\omega(r \ln p)\left(r \in \mathbb{Z}, p\right.$ is a prime number). The groups $G\left(q=p^{r}\right)\left(\operatorname{dim} G\left(q_{1}\right)>\operatorname{dim} G\left(q_{2}\right)\right.$ if $q_{1}>q_{2}$ ) arise. Evidently they contain the Weyl group $W$. In fact the degeneracy of the spectrum is independent of the temperature and symmetries of the systems are not increased. This situation resembles the Dyson description of distributions of levels of heavy nuclei [3]. The distribution of coordinates for the classical twodimensional Coulomb gas for the three temperatures $\beta=1,2,4$ in his approach corresponds to statistical ensembles of orthogonal, unitary and symplectic matrices.

In the case at hand the classical statsum is a Gauss integral which is easily calculated and yields the formula (3.1). It is worthwhile to note that the systems under consideration have nontrivial deformation by adding the potential $g^{2} / x^{2}$ which leads to the class of completely integrable quantum systems of Calogero type. This deformation changes the algebra of creation and annihilation operators but the form of statsums is preserved. The classical statsums are no longer Gauss integrals. Nevertheless it turns out that they are calculable in the quasiclassical limit of the corresponding quantum statsums [17]. As a result one obtains some equivalence between the temperature (or frequency) and group parameters.

3. It is possible also to construct quantum systems related to groups generated by reflections in a complex space $\mathbb{C}^{n}$. In contrast with the relation for a real reflection $S^{2}=1$, in the complex case one has $S^{m}=1$ for an integer $m$. These groups have been classified (see [18]). Moreover as in the real case the CSTT is valid and orders of invariants are listed in [18]. Besides one infinite sequence depending on three integers: $n$ - dim of space, $m$ - an order of reflections and an additional integer number, there exist also 28 exceptional groups in spaces of dimension less than seven. In all the groups the number of semi-invariants is larger than in the real case. It permits to consider quantum systems with various types of conditions (2.9), (2.11). The peculiar feature of complex reflections is the restriction on the smallest order of invariants: $d_{1} \geqq 4$. Recall that in the real case $d_{1}=2$. The most intriguing question which arises here is a possible relation between twenty-six simple sporadic groups and twenty-eight exceptional complex groups.

4. Here we consider another field limit of the theory based on so-called adelic ideology, which first appeared in a physical literature in a connection with the strings and the conformal field theory [19]. To this end it is more convenient to consider fermionic oscillators which are described by the creation $b_{j}^{+}$and annihilation $b_{j}$ operators with the usual anticommutation relations

$$
\left\{b_{j}^{+}, b_{k}\right\}=\omega d_{j} \delta_{j k}
$$

and the Hamiltonian

$$
H_{\text {ferm }}=\frac{1}{2} \sum_{j=1}^{n}\left[b_{j}^{+}, b_{j}\right]+\frac{\omega}{2}(N+n),
$$

where $d_{j}$ are the orders of Weyl invariants. Consider the ground state $|0\rangle: H_{\text {ferm }}$ $|0\rangle=0$. Because the relation $\left[H_{\text {ferm }}, b_{j}^{+}\right]=\omega d_{j} b_{j}^{+}$, we have for the Witten index the 
following expression:

$$
W(p)=\prod_{j=1}^{n}\left(1-p^{-d_{j}}\right), \quad p=e^{\omega},
$$

or comparing it with (1.6) for a prime $p$,

$$
W(p)=\frac{|G(p)|}{p^{\operatorname{dim} \tilde{5}}} .
$$

Now let us consider the ensemble of the fermionic oscillators for all the "prime frequencies"

$$
\omega=\ln p, \quad p=2,3,5,7,11, \ldots .
$$

The Witten index for this ensemble has the form

$$
W=\prod_{p \text {-prime }} W(p) \text {. }
$$

From the Euler formula for the zeta function

$$
\zeta(S)=\prod_{p \text {-prime }}\left(1-p^{-S}\right)^{-1},
$$

the equality $\operatorname{dim}_{n}(\mathfrak{F}=n+2 N$ and (1.3) it follows that

$$
W=\prod_{j=1}^{n} \zeta^{-1}\left(d_{j}\right)
$$

This formula is related to the Tamagawa numbers of adelic groups [20]. We now clarify this relation.

Firstly let us define the Tamagawa numbers.

Adel is the infinite sequence which is parametrized by the prime numbers

$$
a=\left(a_{\infty}, a_{2}, \ldots a_{p}, \ldots\right),
$$

where $a_{\infty}$ is a usual real number with the standard Archimedean norm, $a_{p}$ is a $p$-adic number. The $p$-adic field $Q_{p}$ is the completion of the field $Q$ of the rational numbers by means of $p$-adic norm: $\left|a_{p}\right|_{p}=p^{-r}$, where $r$ is a multiplicity of $p$ in the decomposition of $a$ on prime multipliers. In (3.6) all elements in the sequence after some position are integer $p$-adic numbers. This means that $a_{p} \in \mathbb{Z}_{p}\left(\left|a_{p}\right|_{p} \leqq 1\right)$. The set of sequences (3.6) generates a ring if one defines the addition and the multiplication on their components. From the definition of norms it follows that for any $\lambda \in Q$,

$$
|\lambda|_{\infty} \prod_{p \text {-prime }}|\lambda|_{p}=1
$$

(the product formula).

Consider now an algebraic group $G$ - a subgroup of the linear group $G L_{n}(Q)$ which is defined by polynomial equations. We assume in what follows that $G$ is a semisimple group. For the generic case it is necessary to modify the construction. Let $G_{R}$ be a group of real matrices in $G, G_{p}$ be a group of $p$-adic matrices in $G$ and $U_{p}$ be a subgroup of integer $p$-adic matrices (the matrix elements of $g_{p} \in U_{p}$ are 
integer $p$-adic numbers). The adelic group $G_{A}$ is a set of sequences with the component multiplication

$$
G_{A}=g=\left(g_{\infty}, g_{2} \ldots g_{p} \ldots\right)
$$

where $g_{\infty} \in G_{R}, g_{p} \in G_{p}$, and $g_{q} \in U_{q}$ for $q>p$ for some $p$.

In other words $G_{A}$ is algebraic subset in the product $A^{m}\left(m=n^{2}+1\right)$ which is defined by the same polynomial equations as $G$. The following facts about adelic groups are needed:

1. It is possible to endow $G_{A}$ with a topology in which $G_{A}$ becomes a locally compact topological group.

2. The group over rational numbers $G_{Q}$ is embedded in $G_{A}$ as the diagonal subgroup in (3.8). It is a discrete subgroup in $G_{A}$ which is called the principle adelic subgroup.

3. There exists an invariant measure on $G_{A}$ with a canonical normalization. The construction of this measure deserves a short comment. First of all introduce the highest form $\omega$ on $G$. In local coordinates it takes the form $\omega=f\left(x_{1}, \ldots, x_{m}\right) d x_{1} \ldots d x_{m}$, where $f$ is a rational function with coefficients from $Q$. The invariant measure on $G$ is defined by means of $\omega$ up to the multiplication on $\lambda \in Q$. Similarly one can define forms and measures on $G_{p}(p=\infty, 2, \ldots)$ taking into account the normalization condition $\mu\left(\mathbb{Z}_{p}\right)=1$ for $p=2,3, \ldots$. The invariant measure on $G_{A}$ is the product

$$
\mu\left(G_{A}\right)=\mu\left(G_{\mathbb{R}}\right) \prod_{p \text {-prime }} \mu\left(G_{p}\right) .
$$

The uncertainty in this definition related to the above-mentioned multiplicator $\lambda$ disappears due to (3.7).

4. The coset space $G_{A} / G_{Q}$ has a finite volume which is determined by the invariant measure $\mu\left(G_{A}\right)$ (3.8). It is called the Tamagawa number $\tau(G)$ of a group $G$.

The calculation of $\tau(G)$ is based on the following two statements:

1. The coset $G_{A} / G_{Q}$ is a bundle with the base $G_{\mathbb{R}} / G_{\mathbb{Z}}$, where $G_{\mathbb{Z}}$ is the subgroup of matrices with integer matrix elements and the fibre $U=\prod_{p \text {-prime }} U_{p^{*}}\left(U_{p}\right.$ is a group of integer $p$-adic matrices.)

2.

$$
\int_{U_{p}} \omega_{p}=\frac{|G(p)|}{p^{\operatorname{dim} \mathscr{F}}},
$$

where $|G(p)|$ is the order of the corresponding Chevalley group (1.6).

Therefore

$$
\tau(G)=\operatorname{Vol}\left(G_{\mathbb{R}} / G_{\mathbb{Z}}\right) \prod_{p \text {-prime }} \frac{|G(p)|}{p^{\operatorname{dim} \mathscr{G}}}
$$


Returning to the quantum-mechanical picture we find from (3.3) that

$$
\tau(G)=\operatorname{Vol}\left(G_{\mathbb{R}} / G_{\mathbb{Z}}\right) \prod_{p-\text { prime }} W(p),
$$

where the non-Archimedian part is equal to (3.5). The remaining problem is to include the first multiplier in a definition of the Witten index of a hypothetical quantum system. This multiplier in its turn is proportional to the Witten index $W_{x}$ of the supersymmetric sigma-model with the target-space $X=G_{\mathbb{R}} / G_{\mathbb{Z}}$. In fact the Witten index $W_{x}$ is equal to the Euler characteristic $\chi(X)$ of the model [21]. On the other hand dropping out the subtleties related to the non-compactness of $X$ one finds from the Gauss-Bonnet representation for $\chi$ that $W_{x}$ is proportional to the volume of $X$. This suggests that there should exist a sigma-model partly described by the fermionic Hamiltonians (3.2) in which the Witten index is equal exactly to the Tamagawa number. It should be a topological sigma-model in which only zero modes contribute to the Witten index.

According to A. Weyl's hypothesis $\tau(\widetilde{G})=1$ for universal covering groups $\widetilde{G}$. Now it has been checked in many cases. This equality means that in the hypothetical quantum system the supersymmetry is unbroken. For example, for $S L_{2}$ the formula (3.9) is read as the celebrated Euler relation

$$
1=\frac{\pi^{2}}{6} \prod_{p \text {-prime }}\left(1-p^{2}\right) .
$$

If $G$ is a nonsimply-connected group, $\tau(G) \neq 1$. For example, for $S O_{n}(n \geqq 3)$ $\tau\left(S O_{n}\right)=2$, and therefore in the corresponding system the supersymmetry is broken.

Acknowledgements. I would like to thank A. A. Gerasimov, A. A. Kirillov, Y. I. Kogan, A. I. Morozov, A. M. Perelomov, A. A. Rosly, A. V. Smilga, and A. V. Zelevisnky for illuminating discussions.

\section{References}

1. Schwarz, A.: The partition function of degenerate quadratic functional and Ray-Zinger invariants. Lett. Math. Phys. 2, 247 (1978);

Witten, E.: Supersymmetry and Morse theory. J. Differ. Geom. 17, 661-692 (1982)

Witten, E.: Topological quantum field theory. Commun. Math. Phys. 117, 353 (1988)

Spector, D.: Supersymmetry and the Mobius inversion function. THU 88/41, 1988;

Witten, E.: Quantum field theory and the Jones polynomial. Preprint IASSNS-HEP-88, 1988

2. Gorenstein, D.: Finite simple groups. NY: Plenum Press 1982

3. Dyson, F.J.: Statistical theory of the energy levels of complex systems I, II, III. J. Math. Phys. 3, 140-156, 157-165, 166-175 (1962)

4. Rocha-Caridi, A.: In: Vertex operators in Mathematics and Physics. Lepovsky, J., Mandelstam, S., Singer, I. (eds.). Berlin, Heidelberg, New York: Springer 1985

5. Gross, D.J., Harvey, J.A., Martinec, E., Rohm, R.: Heterotic String theory. Nucl. Phys. 256B, $253(1985)$

6. Gepner, D., Witten, E.: String theory on group manifolds. Nucl. Phys. B 278, 493 (1986)

7. Kac, V.: Infinite dimensional Lie algebras. Cambridge: Cambridge University Press 1985

8. Steinberg, R.: Lectures on Chevalley Groups. Yale University 1967

9. Springer, T.A.: Invariant Theory. Lecture Notes in Mathematics, vol. 585. Berlin, Heidelberg, New York: Springer 1977 
10. Coxeter, H.S.M.: Discrete groups generated by reflections. Ann. Math. 35, 588 (1934)

11. Green, J.A.: Characters of the finite general linear groups. Trans. Am. Math. Soc. 80, 402-477 (1955)

12. Macdonald, I.G.: Symmetric functions and Hall polynomials. Oxford 1979

13. Springer, T.A.: Seminar on algebraic groups and related finite groups. Lecture Notes in Mathematics, vol. 131. Berlin, Heidelberg, New York: Springer 1970

14. Carter, R.: Seminar on algebraic groups and related finite groups. Lecture notes in Mathematics, vol. 131. Berlin, Heidelberg, New York: Springer 1970

15. Kirillov, A.A.: Funct. Anal. Appl. 18, 74-75 (1984) (in Russian)

16. Olshanetsky, M.A.: Wave functions of quantum integrable systems. Theor. and Math. Phys. 57, 148-153 (1983) (in Russian)

17. Olshanetsky, M.A., Perelomov, A.M.: Quantum integrable systems, Phys. Rep. 94, 313-404 (1983)

18. Shephard, G.C., Todd, J.A.: Finite unitary reflection groups. Canad. J. Math. 6, 274-304 (1954)

Cohen, A.M.: Finite complex reflections groups. Ann. Scient. Ec. Norm. Supr. 4, 379-436 (1976)

19. Freund, P.G.O., Witten, E.: Phys. Lett. 199B, 191 (1987);

Manin, Yu.I.: Reflections on arithmetical physics, talk at the Poiana-Brasov school on strings and conformal field theory, 1987;

Witten, E.: Quantum field theory, Grassmannians, and algebraic curves. Commun. Math. Phys. 113, 529-600 (1987)

20. Kneser, M.: In: Algebraic number theory, Cassels, Fröhlich (eds.). New York: Academic Press 1967

21. Witten, E.: Constraints on supersymmetry breaking. Nucl. Phys. B 202, 253-316 (1982)

Communicated by Ya. G. Sinai

Received November 15, 1989 
